\title{
空间可展开天线微重力环境模拟研究现状与展望*
}

\author{
田大可 ${ }^{1}$ 范小东 ${ }^{1}$ 郑夕健 ${ }^{1}$ 刘荣强 $^{2}$ 郭宏伟 $^{2}$ 邓宗全 ${ }^{2}$ \\ (1. 沈阳建筑大学机械工程学院 沈阳 110168; \\ 2. 哈尔滨工业大学机电工程学院 哈尔滨 150001)
}

\begin{abstract}
摘要: 为满足我国深空探测、空间站建设、探月工程等重大航天工程对空间大型可展开天线等新型卫星有效载荷平台快速发 展的要求, 对国内外空间可展开天线微重力环境模拟方法进行了综述。分别对落塔法、拋物线飞行法、水浮法、气浮法和悬 吊法等典型重力补偿方法的工作原理及优缺点进行了分析, 综述了国内外在此领域的应用及技术发展情况, 综合分析并比较 了 5 种微重力模拟方法的技术特点及适用范围, 结合我国航天科技中长期发展规划的需求, 从大型化、高精度化、多样化、 通用化、高保真化等方面, 提出了未来可展开天线微重力模拟的发展趋势及方向, 旨在为我国大型可展开天线及其微重力环 境模拟方法的基础理论研究及工程应用提供借鉴与参考。
\end{abstract}

关键词: 空间可展开天线; 微重力环境模拟; 卸载方法; 微重力水平; 试验系统

中图分类号: V416

\section{Research Status and Prospect of Micro-gravity Environment Simulation for Space Deployable Antenna}

\author{
TIAN Dake $^{1}$ FAN Xiaodong $^{1} \quad$ ZHENG Xijian $^{1} \quad$ LIU Rongqiang $^{2}$ \\ GUO Hongwei $^{2}$ DENG Zongquan ${ }^{2}$
}

(1. School of Mechanical Engineering, Shenyang Jianzhu University, Shenyang 110168;

2. School of Mechanical and Electrical Engineering, Harbin Institute of Technology, Harbin 150001)

\begin{abstract}
In order to meet the requirements of China's deep space exploration, space station construction, lunar exploration and other major aerospace projects for the rapid development of large space deployable antennas and other new satellite payload platforms, the micro-gravity environment simulation methods of space deployable antenna at home and abroad are reviewed. The working principle, advantages and disadvantages of typical gravity compensation methods, such as drop tower method, parabolic flight method, neutral buoyancy method, air floating method and suspension method, are analyzed respectively. The application and technical development in this field at home and abroad are reviewed. The technical characteristics and application scope of five micro-gravity simulation methods are analyzed and compared. Considering the needs of Chinese medium and long-term development plan for space science and technology, and from the aspects of large scale, high precision, diversification, generalization and high fidelity, the development trend and direction of micro-gravity environment simulation for space deployable antenna are proposed. The review provides a reference for the basic theoretical research and engineering application of large deployable antenna and micro-gravity environment simulation methods in China.
\end{abstract}

Key words: deployable antenna; micro-gravity environment simulation; unloading method; micro-gravity level; experimental system

0 前言

空间可展开天线是近二三十年来随着航天科技

* 国家自然科学基金重点(51835002)、中国博士后科学基金面上 (2019M661126)和辽宁省自然基金指导计划(2019-ZD-0655)资助项 目。20200218 收到初稿, 20200722 收到修改稿
的快速发展而产生的一种新型空间结构, 是航天器 的关键有效载荷之一，在卫星通信、军事侦察、深 空探测等领域具有广泛应用并发挥着至关重要的作 用, 已经成为国际宇航界研究的前沿和热点 ${ }^{[1-4]}$ 。

按照天线反射面的结构形式, 空间可展开天线 可分为固体反射面天线、充气反射面天线、金属网 状反射面天线和薄膜反射面天线等四种类型。其中, 
金属网状反射面天线是目前应用最为广泛的一种结 构形式, 主要由柔性金属反射网和可折展刚性支撑 机构组成。空间可展开天线在发射时处于小体积收 拢状态, 收纳于运载火箭的有效载荷舱内, 待卫星 进入轨道后，按控制指令执行解锁-展开-锁定等一 系列动作, 最后展开成大口径空间曲面天线结构 ${ }^{[5]}$ 。 可展开天线在轨工作时处于重力近乎为零的微重力 (大约为 $10^{-4} \mathrm{~g}, \mathrm{~g}$ 为重力加速度) 空间环境中 ${ }^{[6-7]}$, 而 其装配、测试、试验等大量的前期研究工作则需要 在地面环境( $1 \mathrm{~g})$ 中开展。两者较大的重力场环境差 异, 使得地面试验显得尤为关键而重要, 地面试验 的准确性、充分性将直接影响空间可展开天线在轨 工作特性、传输效率、寿命及可靠性等指标参数, 并在一定程度上决定着整个空间任务的成败。因此 为了真实、深入、准确地掌握可展开天线结构在空 间环境的工作特性, 降低空间任务实现的难度和风 险, 需要在地面营造近乎失重的空间环境, 设计相 应的微重力环境模拟试验装置, 开展空间可展开天 线仿真空间环境的试验研究。

发展航天科技是我国中长期科技发展规划的重 要建设内容。为了对以金属网状反射面天线为代表 的空间可展开天线的微重力环境模拟方法和技术有 较为全面的认识和了解, 本文对国内外可展开天线 微重力环境模拟的研究状况进行了概述, 分析了典 型模拟方法的原理、特点及适用范围, 提出了可展 开天线微重力环境模拟试验研究的发展趋势, 旨在 为我国大型可展开天线的基础理论研究及工程实际 应用提供借鉴与参考。

\section{1 国外微重力环境模拟研究现状}

微重力环境模拟的方法从工作原理上, 大致可 以分为运动法和力平衡法两种类型 ${ }^{[8]}$ 。运动法是指 在微重力试验中, 使被测试物体按照某种轨迹进行 运动, 让其自身的重力尽可能全部转化为运动所需 的加速度或离心力等外载荷, 从而消除重力对物体 运动影响的方法。运动法的实现形式主要包括落塔 法、抛物线飞行法、高空气球法和探空火箭法等; 力平衡法是指通过设计某种力平衡装置, 使物体自 身的重力与外力相互抵消, 从而实现微重力模拟的 方法。力平衡法包括水浮法、气浮法、悬吊法和电 磁平衡法等。

本文重点对落塔法、抛物线飞行法、水浮法、 气浮法和悬吊法等 5 种典型的微重力环境模拟方法 进行综述。

\section{1 落塔法}

落塔法 ${ }^{[9]}$ 又称落井法、落管法, 是指采用特殊 的试验装置使具有一定高度的试验舱处于真空状 态, 让物体在舱内执行自由落体运动, 从而使物体 获得微重力状态的方法。试验系统主要由内、外两 个子系统组成, 内部子系统主要包括试验舱和隔离 舱，外部子系统包括落塔的支撑结构、试验舱的升 降机构、减速机构、回收机构及抽真空机构等控制 及操作机构。目前, 美国、日本和德国等国家均建 立了各自的微重力试验系统。

比较有代表性的落塔有: 美国 NASA 的刘易斯 研究中心(Lewis research center, LRC)于 20 世纪 60 年代建立了世界上第一座落塔试验系统, 该系统深 入地下 $155 \mathrm{~m}$, 落差可以达到 $132 \mathrm{~m}$, 微重力时间为 $5.18 \mathrm{~s}$, 微重力水平为 $10^{-5} \mathrm{~g} \sim 10^{-6} \mathrm{~g}$, 平均减速过载 为 $35 \mathrm{~g}$, 峰值过载 $65 g^{[10]}$ 。

日本微重力中心在 20 世纪 90 年代初建成了世 界上最大的微重力落塔试验系统, 如图 1 所示, 该 塔以一个高度为 $710 \mathrm{~m}$ 、直径为 $4.8 \mathrm{~m}$ 的矿井为基础 进行改造, 自由落体高度达到 $490 \mathrm{~m}$, 微重力时间 高达 $10 \mathrm{~s}$, 微重力水平为 $10^{-5} \mathrm{~g}$, 减速过载小于 $10 \mathrm{~g}$, 最大有效载荷重量为 $1000 \mathrm{~kg}$, 有效载荷体积为直 径 $1.3 \mathrm{~m}$, 高度 $1.4 \mathrm{~m}^{[11]}$ 。

德国不莱梅大学应用空间技术和微重力中心 (Center of applied space technology and micro-gravity, ZARM)建立了一个高 $146 \mathrm{~m}$ 的落塔, 如图 2 所示, 该塔可提供 $4.74 \mathrm{~s}$ 的微重力时间, 微重力水平为 $10^{-5} \mathrm{~g} \sim 10^{-6} \mathrm{~g}$, 平均减速过载 $25 \mathrm{~g}$, 峰值过载 $50 g^{[12-13]}$ 。

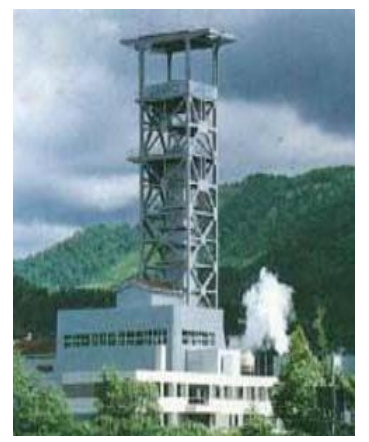

图 1 日本微重力中心落塔

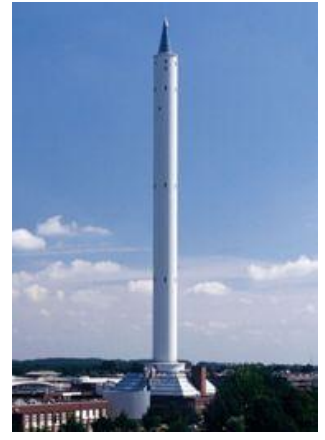

图 2 德国 ZARM 落塔
落塔法的优点是具有较高的模拟精度 $\left(<10^{-5}\right.$ $g$ ), 可模拟三维空间环境, 试验成本低, 可重复性 好, 但落塔的建设成本较高, 试验舱空间有限, 单 次模拟时间较短。

\section{2 抛物线飞行法}

抛物线飞行法 ${ }^{[14-15]}$ 是指将被测试物体安置于对 
燃/滑油系统等适应性改装后的失重飞机内, 让失重 飞机按照抛物线轨迹飞行, 使被测试物体获得微重 力环境 $\left(10^{-2} \mathrm{~g} \sim 10^{-3} \mathrm{~g}\right)$ 的一种方法。失重飞机在进行 抛物线飞行时单次可产生 $20 \mathrm{~s}$ 左右的微重力时间, 并且一次起落可以飞出约 20 40 条抛物线, 累积微
重力时间可达到十几分钟。目前大型的失重飞机主 要有美国的 KC-135-A、俄罗斯的 IL-76MDK、法国 的 Caravelle 6R-234 和 A300B2-100、日本的 MU-300 等几种机型, 表 1 列出了这几种飞机的主要性能参 数 ${ }^{[10,16]}$ 。

表 1 世界主要失重飞机性能比较

\begin{tabular}{|c|c|c|c|c|c|}
\hline 国别与机型 & 俄罗斯 IL-76MDK & 美国 KC-135-A & $\begin{array}{c}\text { 法国 Caravelle } \\
\text { 6R-234 }\end{array}$ & 法国 A300B2-100 & 日本 MU-300 \\
\hline 实验室长 $/ \mathrm{m}$ & 14.2 & 18 & 12.5 & 19.6 & 4.67 \\
\hline 实验室宽 $/ \mathrm{m}$ & 3.45 & 3.25 & 2.7 & 4.9 & 1.5 \\
\hline 实验室高 $/ \mathrm{m}$ & 3.4 & 2 & 1.9 & 2.3 & 1.45 \\
\hline 乘客容量/人数 & 17 & 23 & 21 & 40 & 8 \\
\hline 单次抛物线微重力时间/s & $25 \sim 28$ & 25 & $17 \sim 20$ & $20 \sim 25$ & 30 \\
\hline 一次起落拋物线条数 & $15 \sim 20$ & $20 \sim 30$ & 40 & 40 & 30 \\
\hline
\end{tabular}

2005 年 4 月 5 日, 日本工程实验卫星 (Engineering test satellite-VIII, ETS-VIII) 项目团队在 一架由 A300 飞机改装的失重飞机(ZERO-G)上对 ETS-VIII 天线的部分模块(Large deployable reflector experiment model \#2, LDREX-2)进行了微重力环境 下的展开试验 ${ }^{[17]}$ 。在这次测试中, 飞机在 3 小时的 飞行过程中共飞出了 13 条抛物线。飞行试验现场照 片如图 3 所示。

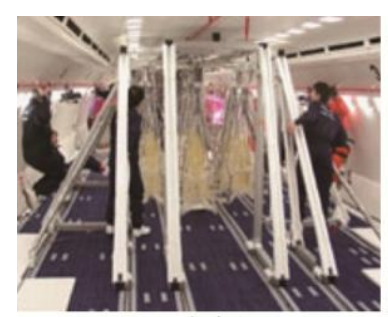

(a) 试验中

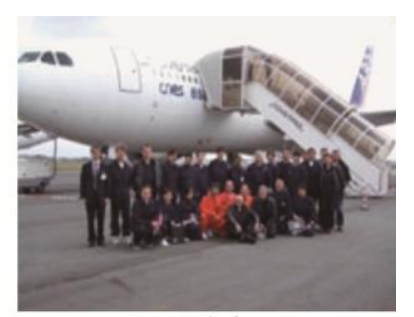

(b) 试验后
图 3 LDREX-2飞行试验

2010 年 3 月, 德国宇航中心(Deutsches Zentrum für Luft-und Raumfahrt, DLR)的 BLOCK 等 ${ }^{[18]}$ 对小型 卫星 AISat 用的螺旋可展开天线在 A300 Zero-G 飞 机上进行了微重力环境下的展开试验, 如图 4 所示, 在这次试验中总共飞出了 31 条抛物线, 每条抛物线 可获得 $22 \mathrm{~s}$ 的失重时间。



(a) 试验照片 1

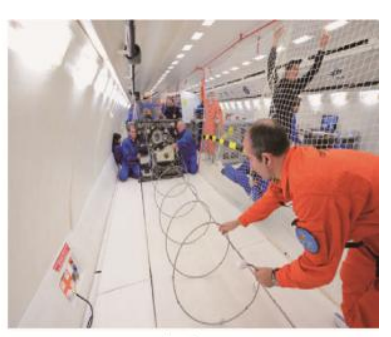

(b) 试验照片2
图 4 DLR 飞行试验
失重飞机的抛物线飞行大致分为 4 个阶段 ${ }^{[15]}$ : 平飞加速阶段、跃升拉起阶段、失重飞行阶段、恢 复平飞阶段。

平飞加速阶段：飞行员控制失重飞机进行抛物 线飞行前的准备, 使失重飞机进行水平加速飞行或 小角度向下加速飞行。跃升拉起阶段：失重飞机调 整飞行速度和姿态等参数, 按预定抛物线轨迹进行 跃升, 飞机仰角在 $40^{\circ} \sim 50^{\circ}$ 。失重飞行阶段：调整 飞机推力, 使飞机的升力与重力近似相等, 飞机进 行失重飞行。恢复平飞阶段: 调整油门杆和飞机姿 态, 将俯冲下降的飞机拉起, 使飞机恢复正常的平 飞状态。图 5 是飞机抛物线飞行产生失重现象的原 理示意图。

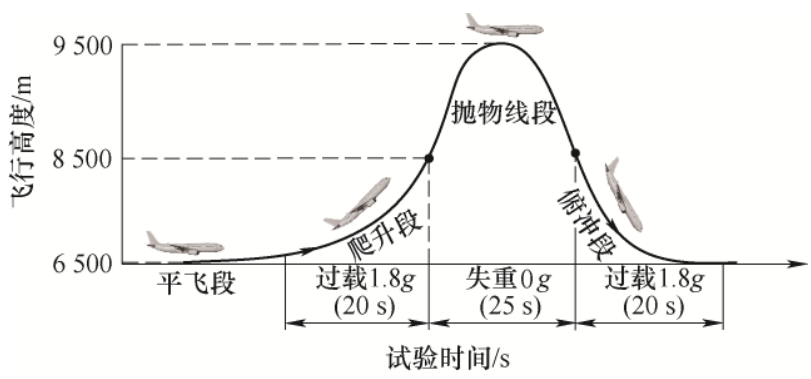

图 5 抛物线飞行示意图

抛物线飞行法可以模拟三维微重力环境, 试验 舱空间较大(平均尺寸约为 $15 \mathrm{~m} \times 3 \mathrm{~m} \times 2 \mathrm{~m}$ ), 主要用 于航天员失重训练, 但模拟精度不高, 单次试验时 间较短, 失重飞机改装成本较大, 加/减速失重飞行 过程中, 载荷变化大, 对飞机的可靠性和安全性有 较大影响。

\section{3 水浮法}

水浮法 ${ }^{[19]}$ 又称为中性浮力模拟法, 是指将被测 试物体经防水、防电等密封特殊处理后, 放入水或 
特殊液体中, 利用漂浮器、液体的浮力等来抵消被 测试物体的重力, 从而实现微重力环境的一种模拟 方法。该方法主要用于对宇航员进行舱内操作、出 舱行走、设备维修、搬运物体等模拟训练以及空间 机械臂 ${ }^{[20-21]}$ 等水下微重力试验。

目前, 比较有代表性的水浮试验系统有俄罗斯加 加林航天员训练中心于 20 世纪 70 年代建立的中性浮 力水槽实验室, 如图 6 所示。该实验室拥有一个尺寸 为 $\varphi 23 \mathrm{~m} \times 12 \mathrm{~m}$ 的圆柱形蓄水池, 容积可以达到 $4983 \mathrm{~m}^{3}$, 实验室可进行舱外活动仪器设备工效学评 价试验、航天员舱外活动训练等试验工作 ${ }^{[22]}$ 。



图 6 俄罗斯水槽实验室

美国拥有 20 余座水下实验室, 为世界之最。美 国的高校、空军、海军及 NASA 等很多科研机构均 建有自己的水下模拟器, 其中, 位于休斯顿的 NASA 约翰逊空间中心建有世界上最大的水下实验室 (Neutral buoyancy lab, NBL), 如图 7 所示。该实验 室为长方形结构, 三维尺寸为 $61.57 \mathrm{~m} \times 31.09 \mathrm{~m} \times$ $12.34 \mathrm{~m}$, 体积达到 $23621 \mathrm{~m}^{3}$, 该实验室可以将国 际空间站模型按 1:1 尺寸放置于水池内, 以便供航 天员进行高保真的模拟训练 ${ }^{[10]}$ 。

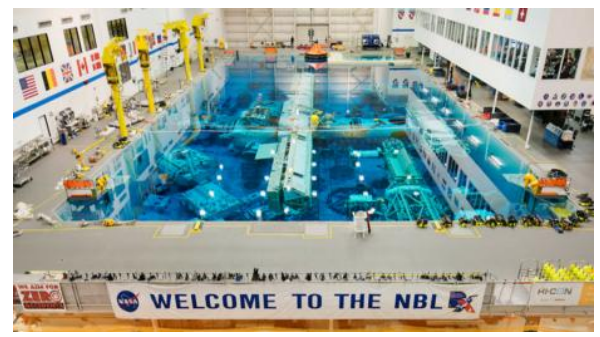

图 7 中性浮力实验室 NBL

美国 NASA 在所建造的中性浮力实验室中进行 了大量的试验, 比较典型的有哈勃望远镜修复的模 拟试验 ${ }^{[23]} 、 大$ 型抛物面精密反射器安装试验 ${ }^{[24]}$ 、大 型空间结构的出舱组装试验 ${ }^{[25]}$ 等。其中, NASA 兰 利研究中心对一个由 315 个零部件组成的口径为 $14 \mathrm{~m}$ 的高精度卫星反射器在水中进行了组装, 整个 安装过程仅用时 $3 \mathrm{~h} 7 \mathrm{~min}^{[24]}$ 。

1995 年, 日本宇宙开发事业集团(National space development agency of Japan, NASDA)在茨城县筑
波市的空间中心(Tsukuba space center, TSC)建立了 一个微重力水下实验室, 该圆筒形水槽的直径为 $16 \mathrm{~m}$, 水深 $10.5 \mathrm{~m}$, 主要用于航天员训练、实验舱 的研制及维护规程的验证等 ${ }^{[22]}$ 。

水浮法的优点是可以实现被测物体较大范围的 三维运动、等比例的模拟试验及较长的试验时间; 缺点是需要对被测试对象进行特殊的密封性处理, 试验设备的维护比较困难, 维护成本高, 同时水流 有黏性及阻力, 为保证模拟的精度, 被测物体仅能 进行较低速率的运动。

\section{4 气浮法}

气浮法 ${ }^{[26-29]}$ 是利用高压气流在光滑的气浮平台 上形成气垫, 将被测试物体托起, 使气体悬浮力与 物体重力相互抵消从而模拟微重力的一种方法。气 浮系统的核心部件主要包括高精度的气浮平台、气 浮轴承、供气系统, 其中气浮轴承等气垫元件需要 进行特殊的设计与制造, 气源通常采用干燥净化的 压缩空气，工作压力一般为 $300 \sim 700 \mathrm{kPa}$ 。

美国斯坦福大学空间机器人实验室拥有多套气 浮试验器 ${ }^{[30]}$, 该试验器主要用于模拟空间机械臂在 轨维护、服务、安装和修理等空间科学任务, 实验 室曾在该试验器上对一个双连杆机械臂的运动包络 空间进行了模拟试验, 机械臂能够在 $1.8 \mathrm{~m} \times 3.6 \mathrm{~m}$ 的气浮平台上自由运动, 如图 8 所示。

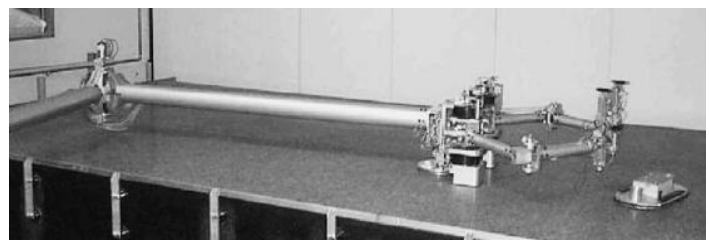

图 8 机械臂运动学试验

日本 NASDA ${ }^{[31]}$ 在其航天项目中针对空间站上 使用的遥控机械臂系统(Japanese experiment module remote manipulator system, JEMRMS), 采用气浮法 对 JEMRMS 的主、副机械臂进行了地面试验, 对机 械臂的定位精度、负载能力、最大运动速度等性能 参数进行了测试, 验证了 JEMRMS 机械臂能够满足 在轨工作的各项技术要求, 如图 9 所示。

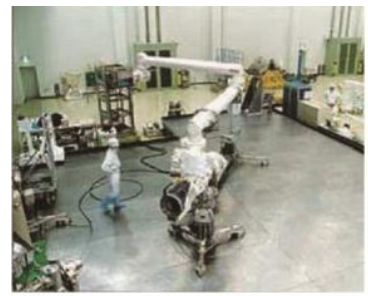

(a) 试验设备调试

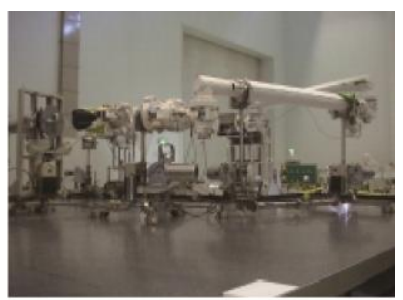

(b) 主臂气浮试验
图 9 JEMRMS 机械臂气浮试验 
气浮法的优点是研制成本低, 摩擦力小, 可进 行较长时间和较高精度的微重力模拟, 对试验件的 体积及重量要求较低, 但辅助系统较为复杂, 主要 用于二维平面状态的微重力模拟, 三维状态模拟技 术尚不成熟。

\section{5 悬吊法}

悬吊法 ${ }^{[32]}$ 是指根据测试物体的具体结构, 选定 合适的吊点位置及吊点数量, 利用吊索拉力平衡被 测试物体重量的一种微重力模拟方法。悬吊法试验 系统一般由支撑框架、拉力装置、随动装置以及控 制系统等组成。按照重力补偿的方式, 悬吊法可分 为主动式和被动式两种, 主动式重力补偿系统主要 是指采用拉力传感器和力矩伺服电动机, 保证吊索 实时保持恒定的拉力; 被动式重力补偿系统主要是 指采用传统的吊丝配重法, 依靠配重块平衡物体的 重力 ${ }^{[33-34]}$ 。

该方法目前在空间可展开天线、机械臂、太阳 翼等航天装备的地面模拟试验中应用较为广泛。比 较典型的应用案例有以下几个。

(1) ASCRL 微重力试验系统。

日本先进空间通信研究实验室 ${ }^{[35-36]}$ (Advanced space communications research laboratory, ASCRL)研 制了一个用于 7 个模块的构架式可展开天线的地面 微重力试验系统, 如图 10 所示。该系统是一种通过 电磁力使滑块悬浮在水平顶板上, 从而消除传统吊 丝配重系统的摩擦力的一种试验系统。该试验系统 由磁悬浮滑块、顶板、电源和控制系统等组成。磁 悬浮滑块在顶板上的水平面内自由移动。水平运动 时的最大阻力小于 $0.1 \mathrm{~N}$, 吊索张力的控制误差小于 $0.25 \mathrm{~N}$, 控制精度小于 $0.5 \%$, 这种系统属于主动重 力补偿系统。

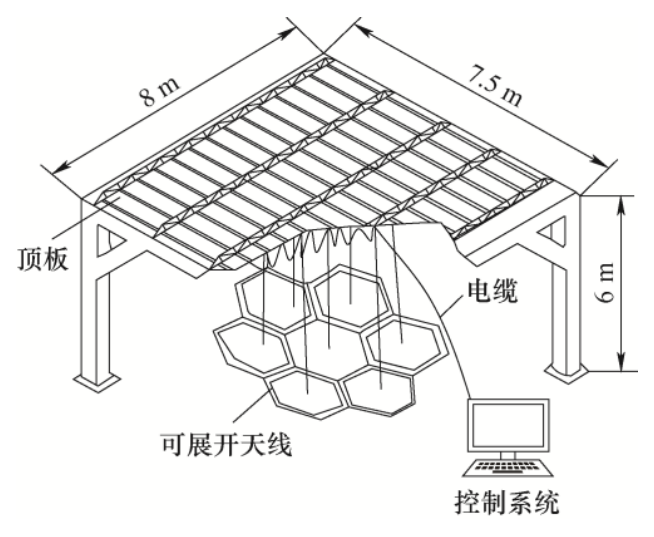

图 10 磁悬浮滑块式悬吊系统

(2) JAXA 微重力试验系统。

日本宇宙航空研究开发机构 ${ }^{[37-38]}$ (Japan aerospace exploration agency, JAXA)对其研制的工
程试验卫星 ETS-VIII 上的大型可展开天线进行了在 轨模拟研究, 设计了一种最大可支持 $14 \mathrm{~m}$ 口径天线 进行地面展开试验的微重力模拟系统, 如图 11 所 示。该系统采用的是吊丝配重法, 每个模块有 6 个 卸载点, 每个卸载点吊索连接的配重块的质量是 $0.5 \mathrm{~kg}$ 。该系统虽然易于实现，但试验过程中的摩擦 和惯性很难克服。

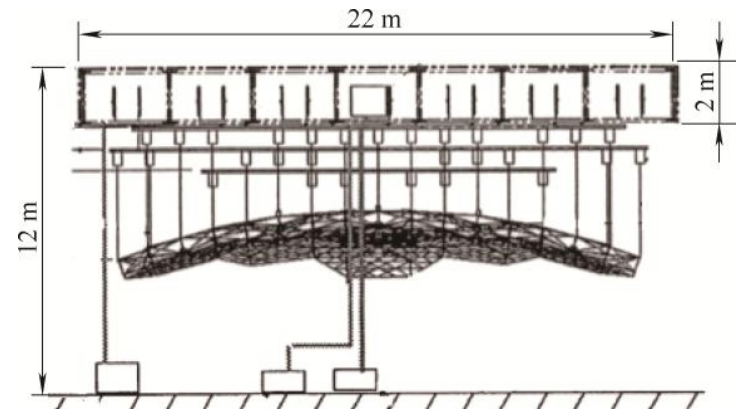

图 11 ETS-VIII反射器展开试验系统

(3) 剑桥大学微重力试验系统。

剑桥大学 FISCHER 等 ${ }^{[39]}$ 为欧洲空间局欧洲可 回收平台(European retrievable carrier, EURECA)上 使用的太阳翼研制了一套微重力试验系统, 如图 12 所示。该系统有 4 个卸载点, 可满足展开长度为 $2.2 \mathrm{~m}$, 质量为 $4.3 \mathrm{~kg}$ 的太阳翼的试验需求; 同时建 立了太阳翼及悬吊系统的力学模型, 对展开过程中 结构的受力情况进行了仿真分析及试验验证。为了 更好的提高悬吊系统的性能、更准确的模拟结构的 动力学特性, 科研团队提出了采用主动式重力补偿 方法和闭环控制算法等改进思路。

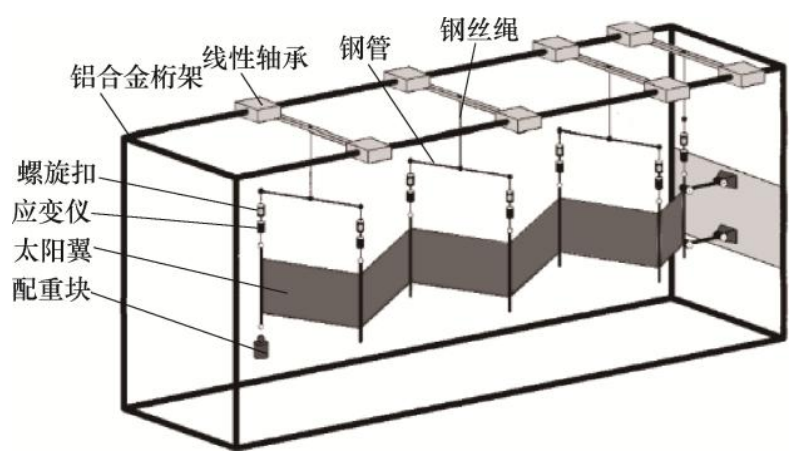

图 12 太阳翼模型和悬吊系统

（4）卡耐基梅隆大学微重力试验系统。

美国卡耐基梅隆大学 ${ }^{[40-42]}$ 针对空间站上使用的 自移动空间机械臂(Self mobile space manipulator, $\mathrm{SM}^{2}$ )先后研制了两种悬吊式微重力试验系统, 两个 系统分别基于笛卡尔坐标系和极坐标系设计, 图 13 所示为改进升级方案, 具有更好的动态响应能力, 系统主要由配重、吊索、电动机、传感器和控制系 统等组成, 能够较好地对机械臂实施重力补偿。 


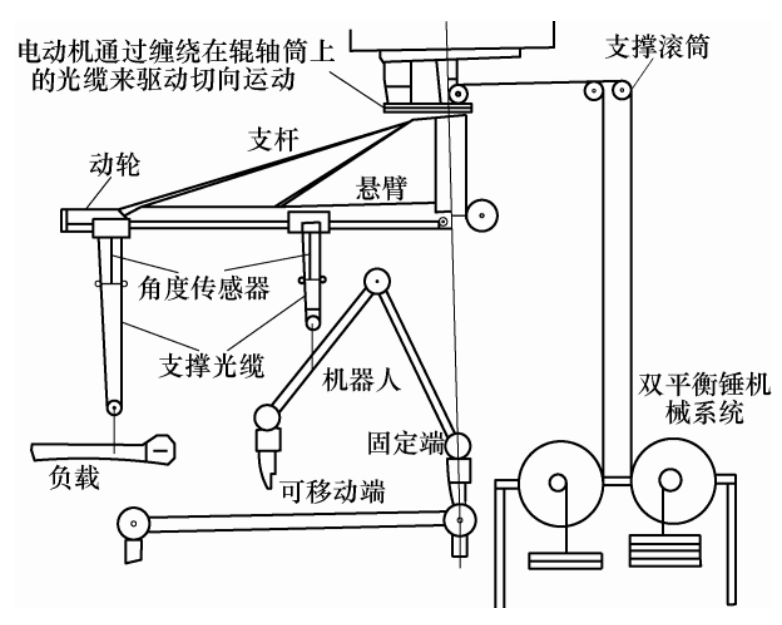

图 $13 \mathrm{SM}^{2}$ 的悬臂试验系统

(5) HALCA 可展开天线的微重力试验系统。

HALCA(Highly advanced laboratory for communications and astronomy)天线是日本宇宙科 学研究所(Institute of space and astronautical science, ISAS)为空间 VLBI(Very long baseline interferometry) 任务设计的一个 $8 \mathrm{~m}$ 口径的可展开天线。为了在地 面验证天线的设计参数和可靠性, 同时设计了一套 地面试验系统, 如图 14 所示, 用 18 个氦气球吊起 金属反射网、每个伸展臂单独使用一个经典的吊丝 配重系统, 总共有 6 个伸展臂, TAKANO 等 ${ }^{[43]}$ 用此 试验系统成功地进行了 9 次试验, 验证了展开的可 靠性。最终，该天线于 1997 年 2 月成功发射。

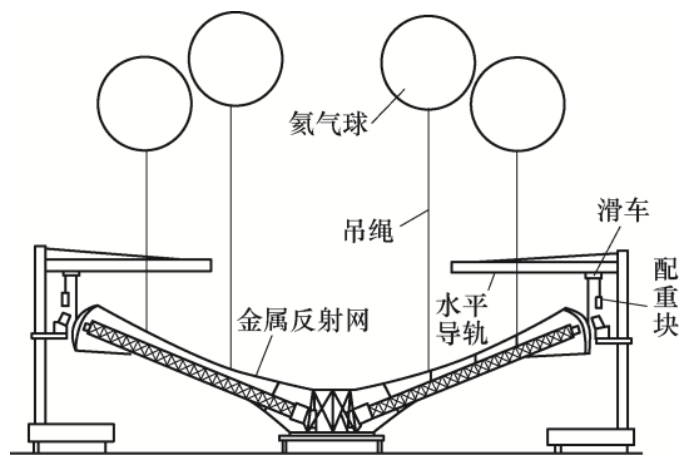

图 14 HALCA 天线试验系统

(6) 欧洲空间技术研究中心微重力试验系统。

MEDZMARIASHVILI 等 ${ }^{[44-45]}$ 设计了一种由 V 型折叠杆构成的雉形环状可展开天线, 如图 15 所 示。为了在地面对可展开天线的设计参数以及可靠 性进行验证, 该团队同时设计了一套悬吊式配重试 验系统, 研制了一个直径为 $6 \mathrm{~m}$ 、质量为 $12 \mathrm{~kg}$ 的样 机, 并在欧洲空间技术研究中心进行了展开试验。

悬吊法的优点是结构易于实现, 可进行三维模 拟, 试验不受场地空间的限制, 试验时间长, 可重 复使用, 应用范围广。缺点是悬吊机构较为复杂,
各构件间存在摩擦阻力, 对测试精度会产生一定程 度的影响。

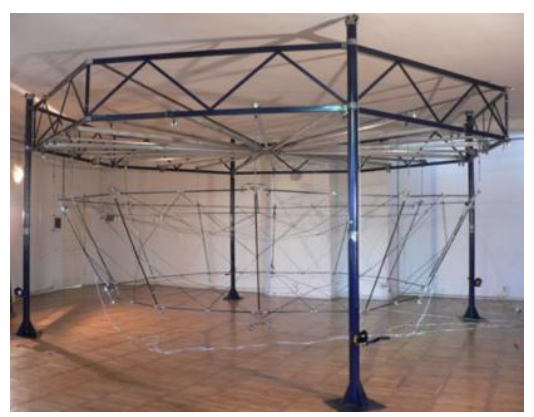

图 15 欧洲空间技术研究中心的重力补偿试验系统

\section{2 国内微重力环境模拟研究现状}

我国的微重力模拟研究起步较晚, 以 20 世纪 90 年代, 原国防科工委(现国防科工局)批准建立的 “国家微重力实验室” 为标志。但经过 20 余年的发 展, 随着我国载人航天、深空探测等空间科学研究 的不断深入，我国的微重力模拟技术取得了长足的 进步和提高。

\section{1 落塔法}

中国科学院 ${ }^{[46-47]}$ 于 2003 年 4 月建成了我国第一 个微重力科学研究中心—国家微重力实验室, 如 图 16 所示。

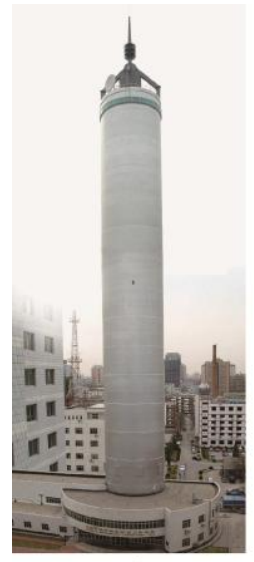

(a) 落塔

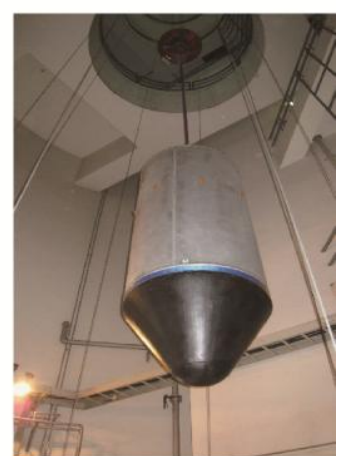

(b) 落舱
图 16 国家微重力实验室落塔

该实验室基于落塔法原理建造，主要包括落塔 和落管等试验设备。落塔高 $116 \mathrm{~m}$, 自由下落高度 为 $60 \mathrm{~m}$, 落塔微重力水平为 $10^{-5} \mathrm{~g}$, 微重力时间为 $3.5 \mathrm{~s}$, 实验载荷质量为 $70 \mathrm{~kg}$, 配有先进的测量、监 测与控制设备。落管自由队落高度为 $45 \mathrm{~m}$, 内径 $200 \mathrm{~mm}$, 微重力时间为 $3.26 \mathrm{~s}$, 微重力水平优于 $10^{-6} \mathrm{~g}$, 配有电阻加热炉、电磁悬浮炉、电子束轰击 炉等。该实验室在我国的载人航天和空间科学技术 
研究等方面发挥了重要的作用。

\section{2 抛物线飞行法}

我国在采用失重飞机进行微重力试验方面公开 报道的文献较少。据原国家宇航局局长、我国首任 “宇航员训练筹备组” 组长薛伦的回忆及相关文献 ${ }^{[48]}$ 记载: 我国曾于 20 世纪 70 年代将一架多-5 飞机改 装成失重飞机, 供我国第一代航天员的训练和选拔, 同时开展其它航空航天试验任务。该飞机为我国载 人航天事业的起步发展立下了难以磨灭的功劳。近 年来, 未见我国在此方向的研究报道。

\section{3 水浮法}

2007 年, 中国航天员科研训练中心 ${ }^{[49-50]}$ 建成了 一个直径为 $23 \mathrm{~m}$, 有效水深 $10 \mathrm{~m}$ 的圆筒形失重训 练水槽, 如图 17 所示。该试验系统主要用于开展航 天员出舱训练、出舱程序验证、航天器设计验证等 相关技术研究。模拟失重训练水槽的主要试验设施 包括: 满足符合航天员和潜水员呼吸用气要求的试 验设备; 2 台升降航天员及仪器设备用岸边吊车; 保证航天员在舒适温度下进行训练的水温调节系 统; 防止科研人员意外跌落水槽的岸边防护栏; 航 天员水下训练应急用供气设备。

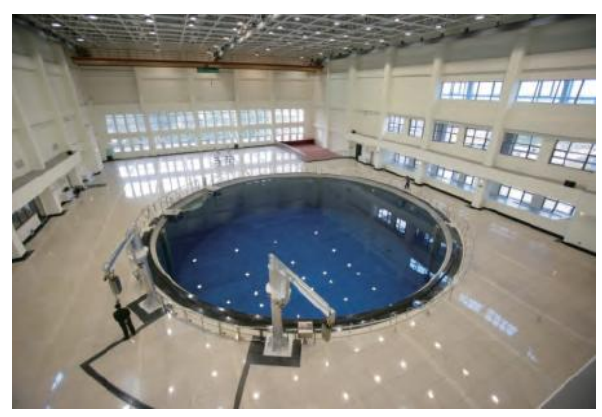

图 17 航天员科研训练中心微重力水槽

西北工业大学航天飞行动力学技术国家重点实 验室 ${ }^{[51]}$ 是以北京航天飞行控制中心为主要依托单 位, 联合西北工业大学成立的国家级重点实验室。 实验室以载人航天、深空探测等重大航天工程为背 景, 通过结合电磁力和水浮力来模拟微重力效应, 建有混合浮力微重力模拟实验系统, 如图 18 所示。

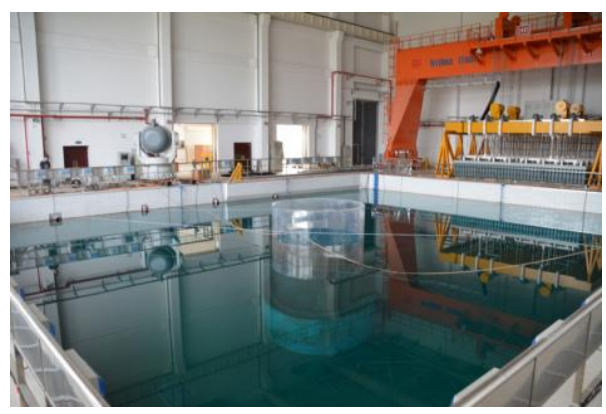

图 18 混合浮力微重力模拟实验系统
该系统被应用于对微型 6 自由度航天器运动的 长时间、大规模、高水平的微重力环境模拟。

\section{4 气浮法}

哈尔滨工业大学气动技术中心是我国较早开展 气浮仿真试验系统研制工作的单位, 建有五自由度 气浮台 ${ }^{[52]}$ 。哈尔滨工业大学齐乃明等 ${ }^{[53]}$ 提出了一种 电动机驱动和气悬浮组合的空间微重力地面模拟装 置, 如图 19 所示。该装置在水平方向采用气垫进行 支撑, 竖直方向采用电动机作为动力执行元件, 采 用滚珠丝杆作为传动元件, 实现三维空间的微重力 模拟，并采用神经网络等控制策略补偿系统中存在 的不确定性影响, 该系统达到了较高的模拟精度。



图 19 三维空间微重力模拟装置

中国科学院沈阳自动化研究所杨国永等 ${ }^{[54-56]}$ 为 了在地面测试中继卫星天线驱动机构的性能, 设计 了一种分级同步重力卸载的气浮试验台, 如图 20 所示。该试验平台采用两层结构的形式, 用气浮垫 和气浮主轴组合实现驱动机构的重力卸载, 并提供 了驱动机构所需的两个关节的转动自由度, 同时对 影响重力卸载精度的因素进行了分析及试验验证。

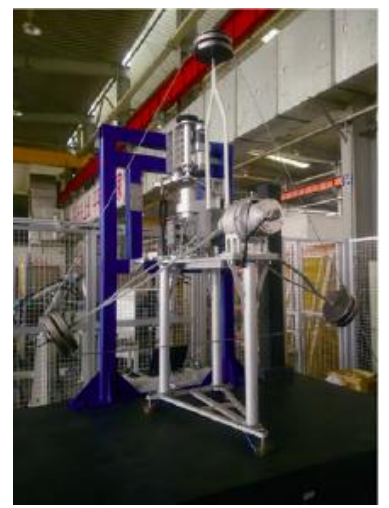

图 20 气浮试验台

北京控制工程研究所与俄罗斯萨玛拉中央专门 设计局进行技术合作，共同研制了大型三轴气浮平 台全物理仿真系统, 并对某大型航天器的单框架控 制力矩陀螺控制系统进行了试验研究, 同时, 开展 了高轨道卫星控制系统全物理仿真试验验证 ${ }^{[57]}$ 。

西北工业大学 ${ }^{[58-59]}$ 以航天器姿态运动为工程背 
景, 以三轴气浮台为物理实验平台, 研究了混沌控 制与反控制问题, 并提出了用外控制力矩进行混沌 化及混沌控制实验的方法。

国防科学技术大学 ${ }^{[60]}$ 对三轴气浮平台进行了 研究, 对总体结构方案进行了设计, 提出了平台的 姿态分析方法, 建立了控制系统仿真模型, 并进行 了初步的单通道试验研究。

\section{5 悬吊法}

浙江大学空间结构研究中心 ${ }^{[61]}$ 在此方向研究 较早, 针对所设计的多种构型可展开天线, 提出了 相应的微重力模拟方案。例如设计了四面体构架式 可展开天线微重力装置, 如图 21a 所示, 该装置采 用中心立柱支撑天线中心节点, 通过斜拉索抵消结 构重力, 并对展开过程中的运动特性进行了试验研 究; 双环可展桁架天线微重力装置, 如图 21b 所示, 该装置利用弹簧绳在内外圈的 24 个节点处进行悬 挂, 用 12 根径向钢管作为天线的支撑结构, 并对展 开口径为 $2 \mathrm{~m}$ 的样机进行了模态试验。



(a) 四面体可展开天线

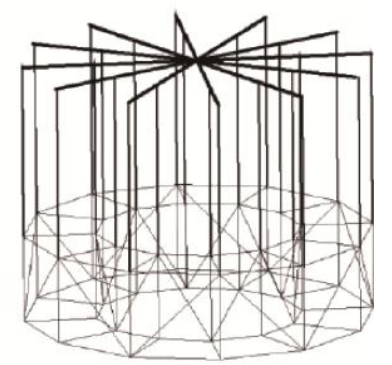

(b) 双环形可展开天线
图 21 四面体及环形可展开天线微重力装置

西安电子科技大学张逸群等 ${ }^{[62]}$ 提出了一种索 网组合式网面可展开天线动力学分析方法, 采用弹 性悬链线单元对松驰、张紧拉索进行了建模, 基于 拉格朗日方程, 建立了可展开天线的柔性多体动力 学模型, 并设计了一套悬吊式微重力试验系统, 如 图 22 所示。

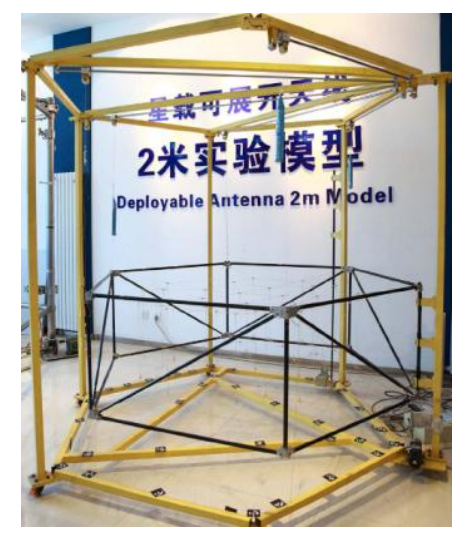

图 22 网面天线重力补偿系统
该系统可满足展开口径 $2 \mathrm{~m}$ 的缩比样机的试验 要求, 在该系统上测试并分析了展开角度与驱动力 的关系, 验证了分析方法的正确性。

哈尔滨工业大学宇航空间机构及控制研究中 心 ${ }^{[63-64]}$ 设计了一种大型空间索杆铰接式伸展臂, 并 研制了相应的悬吊式微重力试验装置, 如图 23 所 示。在该装置上验证了伸展臂及驱动机构的展收功 能, 并对重复展开精度和定位精度进行了测试; 同 时, 该中心还设计了一种模块化构架式可展开天线 地面试验用微重力环境模拟系统 ${ }^{[65]}$, 如图 24 所示, 该系统采用吊丝配重法补偿天线的重力, 并设计了 绳索缓释装置控制天线展开的速度, 实现了天线 7 个模块的同步展开功能验证。

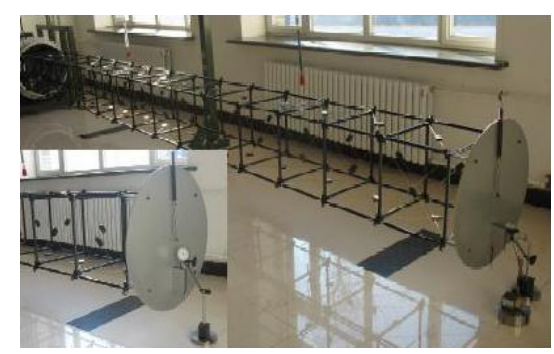

图 23 索杆铰接式伸展臂重力补偿系统

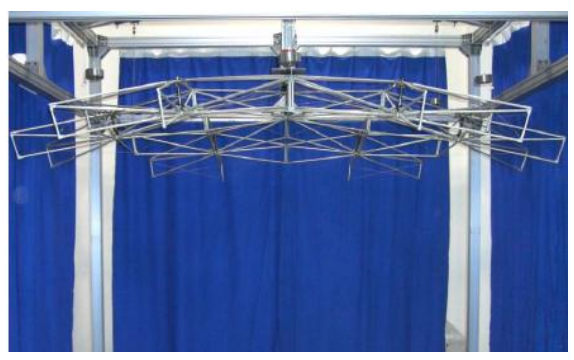

图 24 模块化可展开天线重力补偿系统

中国科学院沈阳自动化研究所贺云等 ${ }^{[66]}$ 为进 行卫星天线展开臂的展开特性测试, 设计了一种卫 星天线展开臂随动吊挂重力补偿系统, 如图 25 所 示。该系统包括 3 轴随动吊挂机械臂、力跟随控制 器及控制系统等装置, 并验证了系统对被测试展开 臂的跟随性能和重力补偿效果。

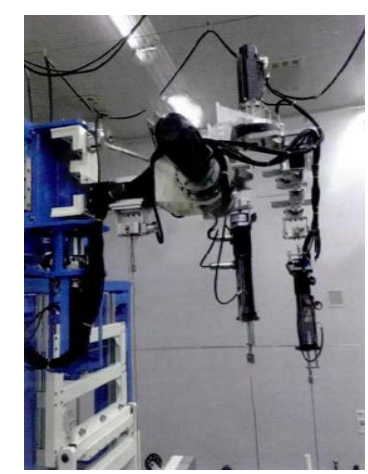

图 25 卫星天线展开臂重力补偿系统 
北京控制工程研究所张新邦等 ${ }^{[67]}$ 对航天器的 系统仿真技术进行了综述, 尤其对航天器机械系统 和姿态控制系统的全物理仿真进行了重点阐述, 并 简要介绍了 “北斗” 导航卫星太阳翼展开试验用悬 吊式微重力系统, 如图 26 所示。

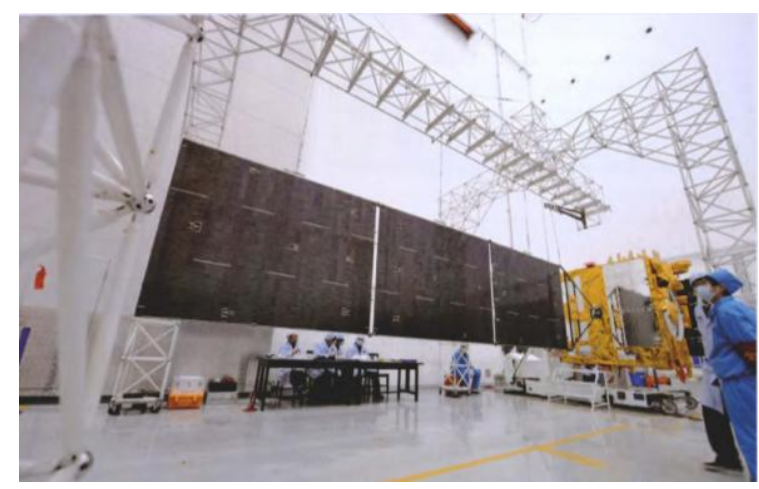

图 26 北斗导航卫星太阳翼展开试验系统

北京卫星制造厂张加波等 ${ }^{[68]}$ 设计了一种太阳 翼重力补偿系统, 该系统通过真空负压吸附力使滑 块吸附在水平顶板上, 从而消除吊丝配重系统的摩 擦力。该试验系统由吸附滑块、顶板、空气压缩机 和控制系统等组成。吸附滑块可在顶板上的水平面 内自由移动, 该系统的摩擦因数能够控制在 0.08 以 下, 吊索在坚直方向的偏差角度不超过 $10^{\circ}$, 在 $40 \mathrm{~N}$ 的工作载荷下, 重力补偿装置的响应误差为 $\pm 5 \%$, 该系统属于被动式重力补偿系统。

北京工业大学张伟等 ${ }^{[69]}$ 为了研究环形桁架式
大型空间可展开天线结构振动模态等动力学特性。 设计并制造了一个环形桁架式可展开天线的缩比样 机, 该样机展开直径为 $1.8 \mathrm{~m}$, 高度为 $0.4 \mathrm{~m}$, 同时 设计了一套实验装置, 在此装置上进行了微重力环 境下的模态试验, 其微重力环境模拟试验系统如 图 27 所示。

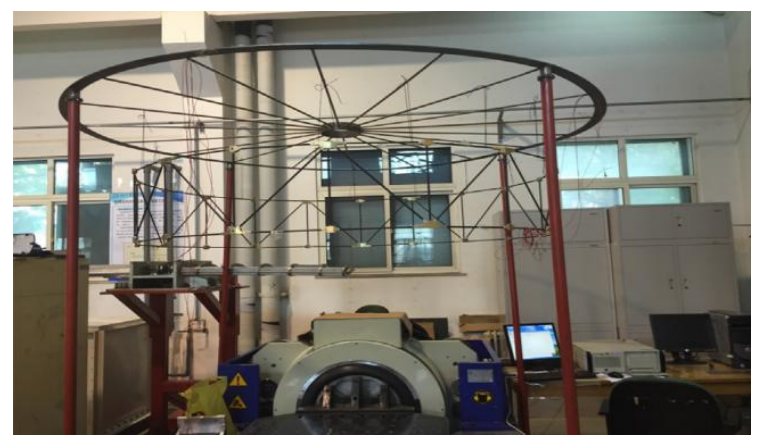

图 27 环形析架式天线重力补偿系统

此外, 中国空间技术研究院西安分院 ${ }^{[70]}$ 、天津 大学 ${ }^{[71]}$ 、清华大学 ${ }^{[72]}$ 、西北工业大学、北京航空航 天大学等研究机构也都提出了很多新的悬吊式可展 开天线重力补偿方案。

\section{3 微重力模拟方法的比较与分析}

综合上述国内外各微重力环境模拟方法 ${ }^{[16,73]}$ 的 原理、特点及优缺点, 可以得到各方法的综合比较 情况，如表 2 所示。

表 2 主要微重力模拟方法参数比较

\begin{tabular}{|c|c|c|c|c|c|}
\hline 模拟方法 & 落塔法 & 抛物线飞行法 & 水浮法 & 气浮法 & 悬吊法 \\
\hline 空间模拟维度 & 3 & 3 & 3 & 2 或 3 & 3 \\
\hline 微重力水平 $/ g$ & $10^{-5} \sim 10^{-6}$ & $10^{-2} \sim 10^{-3}$ & $10^{-1}$ & $10^{-2} \sim 10^{-4}$ & $10^{-2}$ \\
\hline 单次持续时间/s & $\leqslant 10$ & $17 \sim 1000$ & 无限制 & 无限制 & 无限制 \\
\hline 被试件体积/m³ & $<2$ & $<221$ & 取决于水槽尺寸 & 取决于试验台尺寸 & 取决于桁架尺寸 \\
\hline 人为干预 & 间接 & 直接 & 间接 & 间接 & 间接 \\
\hline 试验成本 & 较高 & 高 & 较高 & 较低 & 较低 \\
\hline 难易程度 & 较困难 & 较困难 & 较容易 & 容易 & 较容易 \\
\hline
\end{tabular}

进一步对表 2 中各参数从空间维度、微重力水 平、持续时间、被试件体积等方面比较。

(1) 空间维度。5 种微重力模拟方法均能够对被 试物体进行空间三维模拟, 但气浮法的技术成熟度 相对较低, 应用较少, 在二维模拟方面应用较多。

(2) 微重力水平。落塔法的微重力模拟精度最 高, 微重力水平 $<10^{-5} \mathrm{~g}$, 水浮法的精度最低, 为 $<10^{-1} \mathrm{~g}$, 其余 3 种方法介于两者之间。

（3）持续时间。采用水浮法、气浮法和悬吊法
进行试验时, 可持续的时间最长, 基本可以按照试 验要求持续开展试验研究; 落塔法的时间最短, 小 于 $10 \mathrm{~s}$; 抛物线飞行法介于两类之间。

（4）被试件体积。与持续时间的结论相似, 水 浮法、气浮法和悬吊法可以对体积较大的物体进行 试验; 落塔法受塔身直径的限制, 被试件体积较小; 抛物线飞行法介于之间。

综上, 通过对以上 4 个主要指标及其它方面进 行的比较可见, 在可展开天线微重力模拟方面, 落 
塔法更适用于对可展开天线中体积较小的零部件或 解锁、锁紧等某项关键技术进行试验研究, 由此可 以在较真实的微重力水平下获得较高的试验精度; 水浮法的微重力水平较低, 并且, 由于可展开天线 的杆件和运动副数量众多, 结构较为复杂, 对防水 密封技术提出了很高的要求, 实施方式也非常困难, 同时, 现有计算流体力学 (Computational fluid dynamics, CFD) 难以保证如此复杂结构及系统的模 拟和计算精度; 气浮法由于更多的应用于二维空间 模拟, 尚不适用于具有强三维结构特征的可展开天 线微重力试验; 抛物线飞行法在被试件体积及单次 模拟时间方面偏弱, 目前主要用于宇航员训练, 不 太适用于可展开天线试验; 悬吊法在 5 种典型方法 中各项指标参数更为均衡, 并无明显的弱项, 且易 实施、成本较低, 得到了很多科研机构的青睐, 是 一种较为合适的可展开天线微重力模拟方法, 具有 较大的发展空间和应用潜力。

\section{4 可展开天线微重力模拟的发展趋势}

近年来, 美国、俄罗斯、日本和欧盟等航天大 国和组织, 分别提出了《《国家航天战略》、《20162025 年俄罗斯联邦航天规划》、《第四期中长期发 展规划》(2018-2025 年)和《欧洲航天战略》等航 天战略和规划, 体现了这些国家发展航天科技在其 国防和经济建设中的战略意义。

我国作为航天大国之一，对发展航天科技也始 终保持着高度的重视。2015 年 5 月国务院印发了我 国实施制造强国战略的第一个十年行动纲领 《中国制造 2025》, 纲领提出了我国未来重点突破的 十大发展领域, 其中 “航空航天装备” 领域明确指 出: “发展新型卫星等空间平台与有效载荷、空天地 宽带互联网系统, 形成长期持续稳定的卫星遥感、 通信、导航等空间信息服务能力。” 2016 年 3 月, 我国发布了《中华人民共和国国民经济和社会发展 第十三个五年规划纲要》, 规划提出了未来五年我国 计划实施的 100 个重大工程及项目, “发展新型卫 星等空间平台与有效载荷” 作为一项工程位列其中, 体现了国家的战略布局。由此可见, 作为卫星重要 载荷平台的可展开天线在我国科技发展中具有非常 重要的作用与影响。因此, 为了满足我国经济和社 会对可展开天线的迫切需求, 推动可展开天线领域 的快速发展, 结合前文对国内外微重力模拟方法研 究现状的阐述, 对空间可展开天线微重力模拟方法、 技术和系统等的研究提出以下几点展望。

\section{1 结构尺度大型化}

随着移动通信、对地观测、军事侦察和深空探 测等领域的快速发展, 可展开天线的口径呈大型化 趋势发展, 十米级口径已难以满足发展需求, 数十 米乃至百米级超大口径可展开天线变得愈发迫切。 开展超大口径可展开天线构型创新设计、测试、试 验等技术的预先研究成为了未来的发展方向。

目前, 国内外的微重力系统尚不具备开展此巨 型可展开天线等比例试验的能力。NASA 约翰逊空 间中心的中性浮力实验室虽然三维尺寸较大，但更 多的是开展航天员出舱行走与空间站维护等训练任 务, 尚未有可展开天线整机在水下进行试验研究的 案例, 说明水浮法应用于可展开天线技术不够成熟。 悬吊法在可展开天线试验中应用最多, 系统的研制 成本、周期和模拟精度等综合表现最好，但系统的 尺寸普遍偏小。由此可见, 随着可展开天线大型化 的发展, 微重力模拟系统也应朝着结构尺度的大型 化趋势发展, 尽可能保证地面验证的真实性和准确 性; 同时, 作为技术储备, 还需要在系统的缩放技 术方面开展深入的研究, 提出微重力等效模拟方法, 建立等效模拟仿真模型, 形成缩放技术体系, 发展 缩放模拟系统。

\section{2 卸载及测量高精度化}

随着 $5 \mathrm{G}$ 等新一代移动通信技术的快速发展, 人类对信息的传输量和传输速度提出了更高的要 求。卫星通信的频率也逐渐由 $\mathrm{S}$ 和 $\mathrm{C}$ 频段向 $\mathrm{Ku}$ 和 $\mathrm{Ka}$ 频段提升, 随之而来的是, 要求可展开天线在保 证大口径发展的同时, 其形面精度也要越来越高。 为了更真实的还原可展开天线在轨的工作状态，实 现这一高精度要求, 一个重要的手段是保证地面微 重力模拟系统在卸载及测量方法方面的高精度。

可展开天线通常由众多杆件通过移动副、转动 副等运动副连接而成, 采用电动机、弹簧、记忆合 金等作为动力原件, 采用绳索进行刚化, 是一个典 型的刚柔耦合系统。系统内构件多、运动副多，同 时, 运动副内的间隙及摩擦对结构的运动和受力有 较大影响, 卸载点的位置、数量和分布的确定显得 尤为重要, 需要开展卸载点布局优化研究, 确定合 理的卸载布局方案。同时, 如何布置测量传感器, 采用何种非接触测量方法以尽可能提升测量的准确 性也是微重力模拟系统需要重点解决的问题。

\section{3 试验手段多样化}

具有大容量、多波段、大功率、多功能的卫星 系统已经成为卫星技术未来发展的典型特征。作为 卫星重要有效载荷平台的空间可展开天线, 在完成 
数据传输、数据中继、目标识别、导航定位等多种 任务的同时, 还要经受微重力、高真空、超低温、 强辐射等恶劣的空间环境, 这些都对可展开天线的 可靠性提出了非常高的要求。

为了保证任务实现的成功率, 降低研制风险, 需要在地面开展大量的、充分的试验研究, 而这些 大多以微重力模拟系统为平台, 如开展形面精度测 试、重复展开精度测试、运动特性测试、动力学特 性测试等。但由前述的 5 种典型的微重力模拟方法 可知, 每种方法都有自身的优点, 也都存在一定的 不足, 如悬吊法易实施、易扩展, 但模拟精度低, 落塔法精度高, 但试验时间短。单一的模拟方法无 法胜任可展开天线全部的试验任务。由此可见, 在 对可展开天线进行地面试验研究时, 要根据试验的 内容采取合适的测试方法, 并且要采取多种试验手 段相结合的方式, 充分发挥各方法的优点和长处。

\section{4 试验系统通用化}

空间可展开天线根据工作表面组成介质的不 同, 可以分为四个大的类别, 每个大类又分为若干 个小类, 如金属网面式可展开天线又包括四面体式、 环形析架式、伸展臂式、构架式、折叠肋式等。由 此可见, 可展开天线种类繁多, 形式多样, 这给微 重力系统的设计和研究带来了很大的难度。

目前采取的措施是进行 “个性化” 定制, 根据 所研究的天线设计对应的微重力模拟系统, 这样做 的优点是微重力卸载较为充分、模拟精度高, 但系 统的研制成本高、研制周期长、通用性差, 无法满 足可展开天线快速发展的要求。未来微重力模拟系 统应当具有通用性、可扩展性、装拆灵活性等特点, 在保留天线具体结构个性化的同时, 兼顾其他相近 形式天线的试验要求, 形成一个满足多种天线开展 微重力试验的综合验证平台。

\section{5 模拟研究高保真化}

可展开天线越来越复杂, 研制成本也急剧上升, 一旦在某个关键的微重力模拟环节出现缺失或反复 将对整个科研项目带来不可估量的严重后果。

系统工程是一种对系统内各组成要素进行综合 分析和研究的科学方法, 它的目的是使系统的整体 与局部之间达到协调统一和相互配合, 实现总体的 最优运行。仿真分析是一种将多参数、多变量的复 杂系统进行简化处理, 建立数学模型, 并采用计算 机/计算中心进行虚拟分析的方法, 它的优点是可以 缩短研制时间、降低研制风险和成本。由此可见, 在可展开天线研制的整个过程中, 统筹考虑设计、 制造、试验等各个环节, 开展基于系统工程的全过
程、全三维、高精度仿真分析是可展开天线及其微 重力模拟研究未来发展的一项关键技术。

\section{5 结论}

本文重点对与可展开天线及其所属的空间折展 机构领域相关的 5 种典型微重力模拟方法的国内外 研究进展进行了介绍, 并从空间维度、微重力水平、 持续时间、被试件体积等 4 个方面进行了分析和比 较。结合我国中长期科技发展规划及可展开天线的 应用需求, 对空间可展开天线微重力模拟方法的发 展趋势进行了展望。得到的主要结论如下。

(1) 当前, 我国正处于向经济强国、制造强国 和航天强国迈进的关键期。空间可展开天线作为信 息传递系统的核心装备，在我国探月工程、深空探 测、新型载人飞船和长期在轨空间站等多项重大航 天工程中发挥着至关重要的作用。作为可展开天线 研制关键环节之一的微重力环境模拟同样具有不可 忽视的重要作用。

(2) 从对空间可展开天线模拟的维度、微重力 水平、试验持续的时间等多个指标进行衡量和比较, 目前，水浮法、气浮法和抛物线飞行法应用于空间 可展开天线还有较大的不足和难度; 落塔法较为适 用于对可展开天线的子系统或关键子结构开展试验 研究; 悬吊法则较为全面和均衡, 在应用中具有较 大的潜力和发展空间。

（3）未来，可展开天线将朝着大型化、高精度 化和复杂化方向发展, 同时也将会承担着更为重要 的使命和任务。单一的微重力模拟方法已很难满足 可展开天线的发展需求, 需要不断地对每种方法进 行适应性改进和完善, 同时还要将多种方法进行综 合运用, 达到优势互补, 实现对可展开天线全过程、 全项目、高精度的模拟，从而提高研制成功的概率。

\section{参 考 文 献}

[1] FREELAND R E, BILYEU G D, VEAL G R. Validation of a unique concept for a low-cost, lightweight space-deployable antenna structure[J]. Acta Astronautica, 1995, 35(9-11): 565-572.

[2] MitSUgi $\mathrm{J}$, ANDO $\mathrm{K}$, SENBOKUYA $\mathrm{Y}$, et al. Deployment analysis of large space antenna using flexible multibody dynamics simulation[J]. Acta Astronautica, 2000, 47(1): 19-26.

[3] SONG X K, DENG Z Q, GUO H W. Networking of Bennett linkages and its application on deployable 
parabolic cylindrical antenna[J]. Mechanism and Machine Theory, 2017, 109: 95-125.

[4] ANGELETTI F, GASBARRI P, SABATINI M. Optimal design and robust analysis of a net of active devices for micro-vibration control of an on-orbit large space antenna[J]. Acta Astronautica, 2019, 164: 241-253.

[5] MO L D, CHEN Y K, CHI G X, et al. Experimental study on the multi-dimensional microgravity simulation system for manipulators[C]// 2015 International Conference on Fluid Power and Mechatronics. Harbin, China: IEEE, 2015: 1222-1227.

[6] QIN L, JIA X J, LIU C F, et al. Friction compensation control of space manipulator considering the effects of gravity[C]// The 35th Chinese Control Conference. Chengdu, China: IEEE Computer Society, 2016: 951-956.

[7] QIN L, LIU F C, LIANG L H, et al. Fuzzy adaptive robust control for space robot considering the effect of the gravity[J]. Chinese Journal of Aeronautics, 2014, 27(6): 1562-1570.

[8] 姚燕生. 三维重力补偿方法与空间浮游目标模拟实验 装置研究[D]. 合肥: 中国科学技术大学, 2006.

YAO Yansheng. Research on 3D gravity compensation and equipment of space floating objective simulation[D]. Hefei: University of Science and Technology of China, 2006.

[9] 刘春辉. 微重力落塔试验设备 $[\mathrm{J}]$. 强度与环境, 1993(4): 41-52.

LIU Chunhui. Micro-gravity falling tower test equipment[J]. Structure \& Environment Engineering, 1993(4): 41-52.

[10] 朱战霞, 袁建平. 航天器操作的微重力环境构建 [M]. 北京: 中国宇航出版社, 2013.

ZHU Zhanxia, YUAN Jianping. Construction of microgravity environment for spacecraft operation[M]. Beijing: China Aerospace Press, 2013.

[11] JACK L, ERIC N, RAYMOND S. Capabilities and constraints of NASA's ground-based reduced gravity facilities[C]// The Second International Microgravity Combustion Workshop. Cleveland, Ohio, USA: NASA, 1992: 45-60.

[12] KUFNER E, BLUM J, CALLENS N, et al. ESA's drop tower utilisation activities 2000 to 2011[J]. Microgravity Science and Technology, 2011, 23(4): 409-425.

[13] Center of Applied Space Technology and Microgravity. The Bremen Drop Tower [EB/OL]. [2020-1-4]. https: //www.zarm.uni-bremen.de/de/fallturm/allgemeine-infor mationen.html.

[14] KRAEGER A, PAASSEN R V. Micro- and partial gravity atmospheric flight[C]// AIAA Atmospheric Flight Mechanics Conference and Exhibit. Monterey , California, USA: AIAA, 2002: 1-11.

[15] 屈斌, 王启, 王海平, 等. 失重飞机飞行方法研究 $[J]$. 飞 行力学, 2007, 25(2): 65-71.

QU Bin, WANG Qi, WANG Haiping, et al. Zero-g aircraft flight method research[J]. Flight Dynamics, 2007, 25(2): $65-71$

[16] PLETSER V. Short duration microgravity experiments in physical and life sciences during parabolic flights: The first 30 ESA campaigns[J]. Acta Astronautica, 2004, 55(10): 829-854.

[17] Japan Aerospace Exploration Agency. Engineering test satellite VIII(ETS-VIII)[EB/OL]. [2020-01-04]. https: // global.jaxa.jp/projects/sat/ets8/topics.html.

[18] BLOCK J, BAGER A, BEHRENS J. A self-deploying and self-stabilizing helical antenna for small satellites[J]. Acta Astronautica, 2013, 86: 88-94.

[19] 丁敏. 大跨度伸缩式零重力模拟试验装置设计与分析 [D]. 哈尔滨: 哈尔滨工业大学, 2015.

DING Min. Design and analysis of large span and scalable test device for zeor-gravity simulation[D]. Harbin: Harbin Institute of Technology, 2015.

[20] GEFKE G G, CARIGNAN C R, ROBERTS B J, et al. Ranger telerobotic shuttle experiment: Status report[C]// Proceedings of SPIE-The International Society for Optical Engineering. Newton, MA, United States: SPIE, 2001: 123-132.

[21] CARIGNAN C R, AKIN D L. The reaction stabilization of on-orbit robots[J]. IEEE Control Systems, 2001, 20(6): 19-33.

[22] 成致祥. 中性浮力微重力环境模拟技术 $[\mathrm{J}]$. 航天器环 境工程，2000(1)：1-6.

CHENG Zhixiang. Weightless environment simulation techniques of neutral buoyancy[J]. Spacecraft Environment Engineering, 2000(1): 1-6.

[23] AKIN D, RANNIGER C, DELEVIE M. Development and testing of an EVA simulation system for neutral buoyancy operations[C]// Space Programs and Technologies Conference. Reston, VA, United states: AIAA, 1996: 1-8.

[24] WALTER L, HEARD J, MARK S. Neutral buoyancy 
evaluation of extravehicular activity assembly of a large precision reflector[J]. Journal of Spacecraft and Rockets, 1994, 31(4): 569-577.

[25] ANDERSON D E, JAMES D G, MOORE T O. Using telerobotic operations to increase EVA effectiveness: results of aerobrake assembly neutral buoyancy testing[C]// 4th Annual Conference on Intelligent Robotic Systems for Space Exploration. Troy, NY, United States: IEEE, 1992: 50-60.

[26] SCHWARTZ J L, PECK M A, HALL C D. Historical review of air-bearing spacecraft simulators[J]. Journal of Guidance Control and Dynamic, 2003, 26(4): 513-522.

[27] MENON C, BUSOLO S, COCUZZA S, et al. Issues and solutions for testing free-flying $\operatorname{robots}[\mathrm{J}]$. Acta Astronautica, 2007, 60(12): 957-965.

[28] RYBUS T , SEWERYN K. Planar air-bearing microgravity simulators: Review of applications, existing solutions and design parameters[J]. Acta Astronautica, 2016, 120: 239-259.

[29] CHRISTIAN S. Canadian space robotic activities[J]. Acta Astronautica, 1997, 41(4-10): 239-246.

[30] SCHUBERT H C, HOW J P. Space construction: an experimental testbed to develop enabling technologies $[C] / /$ Proceedings of SPIE - The International Society for Optical Engineering. Pittsburgh, PA, United States: SPIE, 1997: 179-188.

[31] SATO N, WAKABAYASHI Y. JEMRMS design features and topics from testing[C]// 6th International Symposium on Artificial Intelligence, Robotics and Automation in Space: i-SAIRAS 2001. Quebec, Canada: Canadian Space Agency, 2001: 1-7.

[32] SATO Y, EJIRI A, IIDA Y, et al. Micro-G emulation system using constant-tension suspension for a space manipulator[C]// IEEE International Conference on Robotics and Automation. Sacramento, Califomia, USA: IEEE, 1991: 1893-1900.

[33] WHITE G C, XU Y. An active vertical-direction gravity compensation system[J]. IEEE Transactions on Instrumentation and Measurement, 1994, 43(6): 786-792.

[34] MORITA T, KURIBARA F, SHIOZAWA Y, et al. A novel mechanism design for gravity compensation in three dimensional space[C]// IEEE/ASME International Conference on Advanced Intelligent Mechatronics. Kobe, Japan: IEEE, 2003: 163-168.

[35] TSUNODA H, HARIU K, KAWAKAMI Y, et al.
Deployment test methods for a large deployable mesh reflector[J]. Journal of Spacecraft and Rockets, 1997, 34(6): 811-816.

[36] TSUNODA H, HARIU K, KAWAKAMI Y, et al. Structural design and deployment test methods for a large deployable mesh reflector[C]// 38th AIAA/ASME/ASCE/ AHS/ASC Structures, Structural Dynamics, and Materials Conference. Kissimmee, FL, USA: AIAA, 1997: 2963-2971.

[37] MEGURO A, SHINTATE K, USUI M, et al. In-orbit deployment characteristics of large deployable antenna reflector on board engineering test satellite VIIIJJ]. Acta Astronautica, 2009, 65(9): 1306-1316.

[38] MEGURO A, ISHIKAWA H, TSUJIHATA A. Study on ground verification for large deployable modular structures[J]. Journal of Spacecraft and Rockets, 2006, 43(4): 780-787.

[39] FISCHER A, PELLEGRINO S. Interaction between gravity compensation suspension system and deployable structure[J]. Journal of Spacecraft and Rockets, 2000, 37(1): 93-99.

[40] NECHYBA M C, XU Y. Human-robot cooperation in space: $\mathrm{SM}^{2}$ for new space station structure[J]. IEEE Robotics \& Automation Magazine, 1995, 2(4): 4-11.

[41] XU Y, BROWN H B, FRIEDMAN M, et al. Control system of the self-mobile space manipulator[J]. IEEE Transactions on Control Systems Technology, 1994, 2(3): 207-219.

[42] BROWN H B , DOLAN J M. A novel gravity compensation system for space robots[C]// Proceedings of the ASCE specialty conference on robotics for challenging environments (Space94). New York, United States: ASCE, 1994: 250-258.

[43] TAKANO T, NATORI M, MIYOSHI K. Characteristics verification of a deployable onboard antenna of $10 \mathrm{~m}$ maximum diameter[J]. Acta Astronautica, 2002, 51(11): 771-778.

[44] MEDZMARIASHVILI N, MEDZMARIASHVILI E, TSIGNADZE $\mathrm{N}$, et al. Possible options for jointly deploying a ring provided with $\mathrm{V}$-fold bars and a flexible pre-stressed center[J]. CEAS Space Journal, 2013, 5(3-4): 203-210.

[45] PROWALD J S, BAIER H. Advances in deployable structures and surfaces for large apertures in space[J]. CEAS Space Journal，2013， 5(3-4): 89-115. 
[46] National Microgravity Laboratory, Chinese Academy of Sciences, NMLC Overview[EB/OL]. [2020-1-4]. http: // nml.imech.ac.cn/info/detailnewsb.asp?infono=12351.

[47] 张孝谦, 袁龙根, 吴文东, 等. 国家微重力实验室百米 落塔实验设施的几项关键技术 $[\mathrm{J}]$. 中国科学 $\mathrm{E}$ 辑, 2005, 35(5): 523-534.

ZHANG Xiaoqian, YUAN Longgen, WU Wendong, et al. Several key technologies of the national microgravity laboratory's 100-meter drop tower experimental facility[J]. Science in China, Ser. E, 2005, 35(5): 523-534.

[48] 叶介甫. 我国早期筹备宇航员训练始末 $[\mathrm{J}]$. 文史精华, 2011(6): 19-21.

YE Jiefu. The beginning and end of China's early preparations for astronaut training[J]. Literary History, 2011(6): 19-21.

[49] 姚燕生, 梅涛. 空间操作的地面模拟方法一水浮法 [J]. 机械工程学报, 2008, 44(3): 182-188.

YAO Yansheng, MEI Tao. Simulation method of space operation on the ground-buoyancy method[J]. Journal of Mechanical Engineering，2008，44(3): 182-188.

[50] 马爱军, 间利, 徐水红, 等. 国内外典型航天特因环境 选拔训练设备及其应用 [J]. 航天器环境工程, 2019, 36(2): 103-111.

MA Aijun, YAN Li, XU Shuihong, et al. Selection and training equipment for space special environment[J]. Spacecraft Environment Engineering, 2019, 36(2): 103-111.

[51] Northwestern Polytechnical University. Brief introduction of the key laboratory of aerospace dynamics technology [EB/OL]. [2020-01-05]. http : //kypt.nwpu.edu.cn/ index.php?c=content\&a=show \&id=315.

[52] 许剑, 任迪, 杨庆俊, 等. 五自由度气浮仿真试验台的 动力学建模[J]. 宇航学报, 2010, 31(1): 60-64.

XU Jian, REN Di, YANG Qingjun, et al. Dynamic modeling for the 5-dof air bearing spacecraft simulator[J]. Journal of Astronautics, 2010, 31(1): 60-64.

[53] 齐乃明, 张文辉, 高九州, 等. 三维空间微重力地面模 拟试验系统设计[J]. 机械工程学报, 2011, 47(9): 16-20. QI Naiming, ZHANG Wenhui, GAO Jiuzhou, et al. Design of ground simulation test system for three-dimensional spatial microgravity environment[J]. Journal of Mechanical Engineering, 2011，47(9): 16-20.

[54] 杨国永, 王洪光, 姜勇, 等. 气浮试验台重力卸载精度 分析[J]. 机械工程学报, 2019, 55(5): 1-10.

YANG Guoyong, WANG Hongguang, JIANG Yong, et al. Gravity unloading precision analysis of air bearing facility[J]. Journal of Mechanical Engineering, 2019, 55(5): $1-10$

[55] YANG G Y, WANG H G, XIAO J Z, et al. Research on a hierarchical and simultaneous gravity unloading method for antenna pointing mechanism[J]. Mechanical Sciences, 2017, 8(1): 51-63.

[56] YANG G Y, WANG H G, XIAO J Z, et al. Similarity analysis of antenna pointing mechanism running states in space and on the micro-gravity simulator[C]// 6th Annual IEEE International Conference on Cyber Technology in Automation, Control and Intelligent Systems. Chengdu, China: IEEE, 2016: 77-81.

[57] 赵明. 六自由度气浮台控制系统设计[D]. 哈尔滨: 哈尔 滨工业大学，2014.

ZHAO Ming. Control system design of 6-dof airbearing spacecraft simulator[D]. Harbin : Harbin Institute of Technology, 2014.

[58] 孔令云, 周风岐. 用三轴气浮台进行混沌控制与反控制 研究[J]. 宇航学报，2007，28(1): 99-102.

KONG Lingyun, ZHOU Fengqi. A study on the control and anti-control for chaos using 3-axis air bearing table[J] Journal of Astronautics，2007，28(1): 99-102.

[59] 刘荣藏, 周军, 孙剑. 卫星多轴指向姿态控制全物理仿 真实验研究[J]. 宇航学报，2006，27(4): 790-793.

LIU Yingying, ZHOU Jun, SUN Jian. Experiment research for satellites' multi-axis pointing attitude control[J]. Journal of Astronautics, 2006, 27(4): 790-793.

[60] 鲁兴举. 空间飞行器姿态控制仿真试验平台系统研究 与设计[D]. 长沙: 国防科学技术大学, 2005 .

LU Xingju. Research and design of a space craft attitude control simulator system[D]. Changsha : National University of Defense Technology, 2005.

[61] 关富玲, 刘亮. 四面体构架式可展开天线展开过程控制 及测试[J]. 工程设计学报, 2010，17(5): 381-387.

GUAN Fuling, LIU Liang. Deployment control and test of deployable tetrahedral truss antenna[J]. Chinese Journal of Engineering Design, 2010， 17(5): 381-387.

[62] ZHANG Y Q, LI N, YANG G G, et al. Dynamic analysis of the deployment for mesh reflector deployable antennas with the cable-net structure[J]. Acta Astronautica, 2017, 131: 182-189.

[63] 郭宏伟, 刘荣强, 邓宗全. 索杆铰接式伸展臂动力学建 模与分析 [J]. 机械工程学报, 2011，47(9): 66-71. GUO Hongwei, LIU Rongqiang, DENG Zongquan. 
Dynamic modeling and analysis of cable-strut deployable articulated mast[J]. Journal of Mechanical Engineering, 2011, 47(9): 66-71.

[64] 刘荣强, 郭宏伟, 邓宗全. 空间索杆铰接式伸展臂设计 与试验研究[J]. 宇航学报, 2009, 30(1): 315-320.

LIU Rongqiang, GUO Hongwei, DENG Zongquan. Space cable-strut deployable articutlated mast design and experimental study[J]. Journal of Astronautics, 2009, 30(1): 315-320.

[65] 田大可. 模块化空间可展开天线支撑桁架设计与实验 研究[D]. 哈尔滨: 哈尔滨工业大学, 2011.

TIAN Dake. Design and experimental research on truss structure for modular space deployable antenna[D]. Harbin: Harbin Institute of Technology, 2011.

[66] 贺云, 张飞龙, 杨明毅, 等. 卫星天线展开臂的随动吊 挂重力补偿系统设计[J]. 机器人, 2018, 40(3): 377-384. HE Yun, ZHANG Feilong, YANG Mingyi, et al. Design of tracking suspension gravity compensation system for satellite antenna deployable manipulator[J]. Robot, 2018, 40(3): 377-384.

[67] 张新邦, 曾海波, 张锦江, 等. 航天器全物理仿真技术 [J]. 航天控制, 2015，33(5): 72-78.

ZHANG Xinbang, ZENG Haibo, ZHANG Jinjiang, et al. The physical simulation technology for spacecraft[J]. Aerospace Control, 2015，33(5): 72-78.

[68] 张加波, 王辉, 李云, 等. 基于真空负压吸附的太阳翼 重力卸载技术[J]. 机械工程学报, 2020, 56(5): 202-210. ZHANG Jiabo, WANG Hui, LI Yun, et al. Gravity compensation technology of solar array based on vacuum negative pressure adsorption[J]. Journal of Mechanical Engineering, 2020, 56(5): 202-210.
[69] SIRIGULENG B, ZHANG W, LIU T, et al. Vibration modal experiments and modal interactions of a large space deployable antenna with carbon fiber material and ring-truss structure[J]. Engineering Structures, 2020: 1-13.

[70] 苏雯, 杨淑琴, 兰亚鹏, 等. 星载大口径网状天线重力 卸载研究[J]. 机械制造, 2019, 57(6): 67-69.

SU Wen, YANG Shuqin, LAN Yapeng, et al. Research on gravity unloading of spaceborne large diameter net antenna[J]. Machinery, 2019, 57(6): 67-69.

[71] 彭浩, 何柏岩. 星载环形天线重力补偿新方法[J]. 中国 机械工程，2019，30(4): 379-384.

PENG Hao, HE Baiyan. A new gravity compensation method of space-borne perimeter truss deployable reflectors[J]. China Mechanical Engineering, 2019, 30(4): 379-384.

[72] ZHAO Z H, FU K J, LI M, et al. Gravity compensation system of mesh antennas for in-orbit prediction of deployment dynamics[J]. Acta Astronautica, 2020, 167: $1-13$.

[73] SABURO M. Micro-gravity experiments of space robotics and space-used mechanisms at Tokyo institute of technology[J]. Journal of Japan Society of Microgravity Application, 2002, 19(2): 101-105.

作者简介: 田大可, 男, 1981 年出生, 博士, 副教授, 硕士研究生导师。 主要研究方向为空间可展开机构创新设计理论与方法、航空发动机总体 结构及先进技术。

E-mail: tiandake@sjzu.edu.cn

范小东, 男, 1995 年出生, 硕士研究生。主要研究方向为机构学及宇航 空间可展开机构。

E-mail: fanxiaodong@stu.sjzu.edu.cn 郑夕健(通信作者), 男, 1963 年出生, 博士, 教授, 硕士研究生导师。 主要研究方向为宇航空间机构及控制、建筑机械设计与产品开发等。

E-mail: xijianzheng@sina.com 